\title{
Factores socioculturales que influyen en mujeres víctimas de violencia intrafamiliar
}

\section{Sociocultural factors that influence women victims of domestic violence}

\author{
María Mercedes Illescas Zhicay ${ }^{1 *}$, Jenny Irlanda Tapia Segarra ${ }^{2}$ y Elizabeth Teresa Flores Lazo $^{2}$ \\ ${ }^{1}$ Trabajadora social en la empresa Mp3 \\ ${ }^{2}$ Universidad Católica de Cuenca \\ *marym_10_@hotmail.com
}

DOI: https://doi.org/10.26871/killkana_social.v2i3.348

\begin{abstract}
Resumen
La violencia intrafamiliar es un problema de salud física y mental que aqueja a la sociedad en la actualidad, el índice de mujeres que fallecen a manos de sus convivientes ha crecido en el país, los factores determinantes para que este tipo de problema se dé, depende de la cultura y los patrones de crianza que las mujeres tienen desde la etapa de la infancia y el ambiente en el que se desarrollan, las razones por las que las mujeres se ven obligadas a soportar este tipo de maltrato puede ser por el temor que adquieren hacia su pareja, por los hijos o por factores económicos, sin embargo, al vivir bajo estas situaciones también están poniendo en riesgo su vida y la de sus hijos y predisponiendo a que estos estén propensos a desarrollar actitudes similares a la de sus padres, volviéndose este un problema trasmitido de generación en generación. Determinar los factores socioculturales que influyen en mujeres víctimas de violencia intrafamiliar será indispensable para la intervención desde el trabajo social, se ha considerado como objeto de estudio a mujeres que acuden a los centros de apoyo a la mujer en el cantón Cuenca provincial del Azuay. Estudios realizados por la OPS (Organización Panamericana de la Salud) y la OMS (Organización Mundial de la Salud), en el 2000, se identifica a diez países latinoamericanos entre los cuales figuran: Belice, Bolivia Costa Rica, Ecuador, El Salvador, Guatemala, Honduras, Nicaragua, Panamá y Perú, establecen que la mayoría de actos de violencia contra la mujer se dan debido al ambiente en el que se desarrollan.
\end{abstract}

Palabras clave: Violencia Intrafamiliar, cultura, factores socioculturales, familia.

\begin{abstract}
The domestic violence is a problem of physical and mental health that afflicts society today, the rate of women who die at the hands of their cohabitants has grown in the country, the determining factors for this type of problem is given, depends of the culture and parenting patterns that women have from the childhood stage and the environment in which they develop, the reasons why women are forced to endure this type of abuse can be due to the fear they acquire. towards their partner, children or economic factors, however, living under these situations are also putting their lives and their children's lives at risk and predisposing them to be prone to developing attitudes similar to their parents', turning This is a problem transmitted from generation to generation. To determine the sociocultural factors that influence women victims of intrafamily violence will be essential for the intervention from social work, it has been considered as object of study to women who go to the support centers for women in the canton Cuenca del Azuay. Studies conducted by PAHO (Pan American Health Organization) and WHO (World Health Organization), in 2000, identifies ten Latin American countries, which include: Belize, Bolivia, Costa Rica, Ecuador, El Salvador, Guatemala, Honduras, Nicaragua, Panama and Peru, establish that the majority of acts of violence against women occur due to the environment in which they develop.
\end{abstract}

Key words: Intrafamily violence, culture, sociocultural factors, family.

\section{Introducción}

La violencia intrafamiliar en América Latina, se ha constituido en un grave problema a nivel social y de salud, estudios realizados por la OPS (Organización Panamericana de la Salud) y la OMS (Organización Mundial de la Salud) en el 2000, son diez países de Latinoamérica entre los cuales figuran: Belice, Bolivia Costa Rica, Ecuador, El Salvador, Guatemala, Honduras, Nicaragua, Panamá y Perú, establecen que la mayoría de actos de violencia contra la mujer se dan debido al ambiente en el que se desarrollan ya sean por patrones culturales, tradicionales, sociales o religiosos, de acuerdo a la investigación en los centros de apoyo a la mujer en el cantón Cuenca, las mujeres se ven 
obligadas a callar este tipo de abuso, por miedo a la desprotección, y por la escasa respuesta a nivel social, por lo que exigen una legislación apropiada que protejan sus derechos, y servicios que sean eficaces que promuevan una sociedad igualitaria y justa. De esta manera se hace referencia a la "Necesidad de un cambio cultural mayor que descontruya las relaciones básicas de poder y las jerarquías sociales, a fin de construir una sociedad más justa, igualitaria y libre de violencia para las mujeres y otros grupos subordinados". (Sagot, 2000).

Estudio realizado en Ecuador sobre la violencia de género establece que 6 de cada10 mujeres han sido víctimas de algún tipo de violencia, existen diferentes factores que ubican a las mujeres en mayor condición de vulnerabilidad como aquellas que tienen algún tipo de discapacidad, por ser indígenas o aquellas que son miembros de hogares con bajos recursos económicos, de la misma manera expresan que aquellas mujeres que tienen un nivel alto de educación son menos propensas a sufrir este tipo de maltratos, sin embargo no las excluye de que alguna vez puedan ser sometidas a los mismos. "Se trata de la confluencia de múltiples discriminaciones: por ser pobre, por su condición de género, su pertenencia étnico-cultural y por su discapacidad" (Quintana Zurita, Serrano Salgado, Pimentel Bolaños, y Moncayo, 2014).

Por lo tanto, se puede manifestar que los agresores están en diferentes lugares de la sociedad tales como: escuelas, colegios, barrios, medios de transporte, parques, centros de diversión, se puede considerar que la mayor parte de ellos se encuentra dentro del propio grupo familiar. A pesar que en el Ecuador se haya avanzado con la tipificación de este delito, aún persisten nociones tradicionales en cuanto a género y desvalorización de la mujer, esto se puede evidenciar en diferentes contextos sociales como lo puede ser el lugar de trabajo o de estudios, la desigual distribución de poder y la asignación de roles estereotipados o infundados por la propia sociedad en la que se desenvuelve la persona, sin dejar de lado la cultura patriarcal en la que las mujeres se han desarrollado desde la niñez.

El presente estudio tiene como finalidad determinar los factores socioculturales que influyen en mujeres víctimas de violencia intrafamiliar en los servicios de apoyo a la mujer del cantón Cuenca puesto que la Convención sobre la eliminación de todas las formas de discriminación contra la mujer "Reconoce el papel de la cultura y las tradiciones en el mantenimiento de la discriminación contra las mujeres, y obliga a los Estados a eliminar los estereotipos en los roles de hombres y mujeres" (Convención sobre la eliminación de todas las formas de discriminación contra la mujer , 2010), lo que implica establecer los principales factores culturales que predominan en la actualidad dentro de una cultura machista, con miras a una sociedad más igualitaria que respete los derechos establecidos entre hombres $\mathrm{y}$ mujeres.

El estudio se abordará desde el tipo de investigación mixta no experimental, transversal durante el periodo Mar- zo -Agosto del 2017, se aplicó el método cuali-cuantitativo, en este caso el estudio cualitativo se ver reflejado directamente con entrevistas a las usuarias en donde se les explicó el motivo de la investigación a la vez que se les comunico que la información que otorguen será de absoluta confidencialidad y que tienen derecho a no participar de este estudio. Mientras que para el estudio cuantitativo se aplicó un cuestionario previamente establecido en donde constaron preguntas acerca de la cultura familiar de edad del usuario, grupo familiar, factores relacionados con la familia de origen, violencia intrafamiliar, escolaridad entre otros, que en correlación con la información dieron los resultados con mayor credibilidad y capacidad de explicación de los mismos. La investigación permitió desarrollar nuevas destrezas logrando comprender de manera amplia y profunda el fenómeno a estudiar, será de carácter descriptiva, por tanto, busca narrar los factores socioculturales que influyen en mujeres que han sufrido violencia intrafamiliar.

\subsection{Factores culturales}

La cultura en la que se ha desarrollado la mujer, desde su niñez ha influenciado de manera directa en su comportamiento y manera de pensar en la actualidad, el hecho de vivir en una sociedad en la que aún persiste el machismo ha constituido uno de los mayores problemas de violencia contra la mujer en los últimos tiempos. El Plan Nacional de Erradicación de la Violencia de Género (2007) expresa:

La cultura está conformada por valores, prácticas, relaciones de poder y proceso sociales, políticos y económicos, que se entrelazan en la vida cotidiana de las personas y de las comunidades creando patrones culturales que no son de manera alguna un conjunto estático y cerrado de creencias y prácticas. En este conjunto de creencias y prácticas, de patrones culturales, que fluyen y cambian llamado cultura, existen actitudes o conductas que perpetúan y generan prácticas que entrañan violencia o coacción basada en género, puesto que se (re)producen en la atribución de funciones estereotipadas a hombres (niños, jóvenes y adultos) y mujeres (niñas, jóvenes y adultas) en las que se considera a niños, niñas adolescentes y mujeres, como subordinados.

De manera que los estereotipos y roles expuestos para cada sexo influyen de manera directa durante el crecimiento tanto de la mujer como del hombre, dependiendo del ambiente en el que se desarrolla, los valores que le han sido inculcados desde la infancia definirán su personalidad y actitud en su vida adulta. Las creencias idealizadas que se tienen del poder superior del hombre han constituido un obstáculo a nivel general para el desarrollo de la mujer y ha perpetuado la violencia dentro del hogar, constituyéndose en un problema a nivel social y de salud pública.

Resaltando lo señalado en los párrafos anteriores del Plan Nacional de Erradicación de la Violencia de Género expone:

Algunas de estas actitudes o creencias tienen que ver con la serie de mitos que (re) producen la 
creencia en la superioridad del sexo masculino, que resulta en una serie de privilegios. La más extendida es la hipernecesidad sexual masculina que está relacionada al desempeño sexual para determinar la mayor o menor hombría. La explotación sexual de niños, niñas y adolescentes, así como la prostitución forzada de mujeres adultas, encuentran en esta creencia una fuente de naturalización. "La violencia sexual es también más frecuente allí donde impera un firme convencimiento de los derechos sexuales del varón o los roles de los sexos son rígidos. (Presidente Constitucional de la República, 2007).

En la actualidad se evidencia que desde la niñez los padres de familia asignan diferentes actividades para niños y niñas que van desde su forma de vestir hasta los juegos que puede establecerse dependiendo de su sexo, lo que crea una brecha enorme porque en sí son las mujeres las principales fuentes de enseñanza de los hijos sin menoscabar la responsabilidades de los padres, sin embargo las madres educan a las niñas como personas frágiles y con actividades de menor peso que los niños, mientras tanto en los varones lo que hacen es inducir un carácter fuerte, con ideas claramente machistas por lo que a través del tiempo estas se incorporan a su vida cotidiana transformándose en estereotipos, actitudes y creencias que se van trasmitiendo.

Una de las causas frecuentes por las que las mujeres son víctimas de violencia son los patrones culturales que se han trasmitido a lo largo de su vida en el que se minimiza el papel de la mujer y prevalece la subordinación ante los hombres, como lo dice un estudio realizado en España al hablar de los factores socioculturales: "Este tipo de violencia no es el resultado de casos inexplicables de conducta desviada o patológica. Por el contrario, es una práctica aprendida, consciente y orientada, producto de una organización social estructurada sobre la base de la desigualdad entre hombres y mujeres" (Ruiz-Perez, BlancoPrieto, y Vives-Cases, 2004).

\subsection{Mitos y Creencias}

Según Labrador, Paz, Luis \& Fernández (2004), nombran diferentes creencias que perduran en la sociedad en cuanto a la violencia intrafamiliar:

- La violencia es un problema de clases sociales bajas. - El maltrato doméstico es producto de algún tipo de enfermedad mental.-El abuso de drogas y alcohol es responsable de violencia doméstica.-Las víctimas de maltrato a veces se lo buscan, hacen algo para provocarlo.-Las mujeres que son maltratadas permanecen en esa situación porque les gusta.-Si una mujer maltratada dejara a su marido, estaría a salvo.- La mayoría de las víctimas de violencia doméstica no sufren heridas de gravedad.- El maltrato psicológico no es tan grave como el maltrato físico.-La conducta violenta es algo innato, que pertenece a la esencia del ser humano.
- Del contexto expresado, se pueden evidenciar las creencias que aún persisten dentro de la sociedad, en la que las mujeres han sido las víctimas más frecuentes de violencia intrafamiliar. Este problema no solo se da dentro de las clases sociales bajas, simplemente es en este sector de la sociedad más visible, no se puede asumir que el consumo de alcohol sea una causa para que se de la violencia, como tampoco se puede decir que la mujer que permanece con su maltratador sea porque le guste, debido a que existen diferentes motivos por los cuales las mujeres decidan soportar aquello, entre ellos están los hijos, el miedo y las amenazas de la persona maltratadora, entre otras, la violencia psicológica muchas veces es más grave que la física, pues las personas pueden llegar a caer en un estado de depresión poniendo en riesgo su vida.

Algunos mitos en cuanto a las causas de violencia intrafamiliar según Soledispa Toro \& Garbay Mancheno (2004) expresan:

Los trapos sucios se lavan en casa. -Se sostiene que el problema se debe a la vigencia de usos y costumbres; por lo tanto, es natural que en la vida de toda pareja haya violencia, pero ésta es controlable. - Se prioriza la necesidad de conservar la "unidad" familiar a como dé lugar: "una familia con padre y madre, aunque sea malavenida, es siempre mejor que una familia sin padre".

Estos patrones de pensamientos que han trasmitidos de generación en generación han repercutido de manera negativa para el desarrollo de las mujeres, pues en muchos casos, el miedo a denunciar actos de violencia, las obliga a permanecer en situaciones de desventaja frente a la pareja, la mayoría de las mujeres consideran que es su deber mantener la unidad familiar a pesar de la violencia que ejercen contra ellas, otras naturalizan a la violencia como algo normal de pareja y perduran años con su maltratador.

\subsection{El androcentrismo y patriarcado}

Según el Equipo Técnico del Departamento de Planificación y Gestión por la Equidad Social y de Género del Cantón Cuenca (2016), al hablar de androcentrismo expone que: "Es la visión del mundo que sitúa al hombre como centro de todas las cosas, desde una posición que generaliza las experiencias masculinas universales", creando un sistema en donde presenta al hombre como un modelo a seguir y que las mujeres deben ajustarse a ese estándar y consecuentemente seguir los pasos marcados por los hombres. Lo que invisibiliza la opinión y las necesidades de las mujeres en la sociedad, cayendo en la subordinación de los hombres, por cuanto estos ejercen poder sobre ellas e imponen condiciones propias del patriarcado.

El sistema patriarcal viene dado desde la cultura primitiva, por hechos o circunstancias que se originaron desde el principio de la vida, en donde el hombre por su fortaleza era quien salía en busca de alimentos, mientras tanto la mujer por su condición era tomada como un ser inútil, que únicamente debía cuidar a sus hijos, este sistema patriarcal 
se ha ido fortaleciendo con el pasar del tiempo, a la vez que ha sido trasmitido de generación en generación, lo cual resulta un tanto difícil cambiar estas ideas y cultura. Según Alda Facio, el patriarcado es: "La manifestación del domino masculino sobre las mujeres y los/las niños/as de la familia, que se extiende a la sociedad en general" (Equipo del departamento de planificación y gestión para la equidad social y de Género, 2016). Lo que explica que una persona que nace en un ambiente en donde el hombre es sinónimo de poder y esclaviza a la mujer puede llegar a desarrollar este mismo comportamiento en su vida adulta, propagando la violencia y sometimiento a la mujer.

\subsection{Sexo y género}

Al hablar de sexo y género en el presente trabajo de investigación, lo que se pretende es realizar una diferencia profunda de estos dos términos y su relación ligada a la discriminación y violencia que en esta se genera, el sexo hace referencia a: "Diferencias biológicas entre hombres y mujeres" (Equipo del departamento de planificación y gestión para la equidad social y de Género, 2016), en cuanto a sus órganos genitales, y a la función que cumple cada uno en la preservación de la especie humana y el desarrollo del hombre, es desde allí en donde parten los modelos que caracterizan y diferencian a lo femenino de lo masculino, de esta manera se emprenderá una educación diferenciada en donde tanto mujeres como hombres adquirirán diferentes actitudes, pensamientos y comportamientos dependiendo el ambiente en el que se encuentre y el grupo familiar en el que haya nacido.

Cuando se trata de género se hace referencia a: "Una categoría que nos permite analizar las relaciones sociales, culturales, políticas, familiares que se dan entre mujeres y hombres; entender que estas relaciones son socialmente construidas y definen roles diferentes para hombres y para mujeres" (Equipo del departamento de planificación y gestión para la equidad social y de Género, 2016), por lo que se entiende que el género se aprende de acuerdo a la sociedad en donde se desarrolle, sin embargo es aquí en donde se crea una brecha enorme de desigualdad e inequidad entre hombres y mujeres pues se establecen roles diferenciados y propios de cada sexo, nombrando a la mujer como el sexo débil, a la vez asumiendo responsabilidades únicas como la educación de los hijos, el servir únicamente para ser ama de casa mientras los hombres son llamados jefes de hogar y los responsables económicamente de sustentarlo.

También se encuentran los estereotipos de acuerdo al género que: "Son ideas arraigadas que determinan las conductas, comportamientos y actitudes que deben tener tanto mujeres como hombres y que por lo general, son opuestos" (Equipo del departamento de planificación y gestión para la equidad social y de Género, 2016), por lo que a las mujeres se les toma como personas subordinadas, de sumisión, objeto sexual, dependencia etc., mientras que a los hombres se les asigna estereotipos de poder, razón, violencia, dominación y un ser independiente propias de una sociedad patriarcal que perdura en nuestro medio: "Las desigualdades de género así como la estigmatización de la diversidad sexual son prácticas constantes que forman parte de las estructuras patriarcales de la sociedad cuencana”. (Municipalidad de Cuenca, 2015).

\subsection{Relaciones de género}

Las relaciones de género según Soledispa Toro \& Garbay Mancheno (2004), son aquellas que: "Se dan entre mujeres y hombres: de igualdad o de desigualdad; y, que son el resultado de ideas, costumbres y creencias de cada sociedad". Como se expresa, son relaciones que se construyen en la sociedad de acuerdo a su cultura y costumbres, a pesar de ello estas pueden perdurar o modificarse al pasar del tiempo. En la actualidad se ha buscado generar una igualdad de género, sin embargo, en una sociedad machista es difícil erradicar o tratar de cambiar esquemas que han sido impuestos en la sociedad desde la infancia hasta la adultez, de tal manera es preciso educar a los hijos desde su infancia en normas y fomentando el respeto e igualdad entre hombres y mujeres.

\subsection{Las relaciones familiares}

Dentro de estos encontramos en primer lugar a la familia, pues en ella se generan los primeros patrones de comportamiento de una persona, luego está el barrio en el que vive la persona y la sociedad en general: "El modelo de aprendizaje social postula que la conducta violenta es aprendida en el hogar, cuyos miembros la repiten posteriormente cuando forman sus propias familias". (Espinoza Morales, Alazales Javiqué, Madrazo Hernández, García Socarrás, y Presno Lavrador, 2001). Lo que expresa que si un niño crece en un hogar en donde existe constante violencia, este podrá desarrollar estos comportamientos a lo largo de su vida, al contrario de las niñas que pueden llegar a sufrir maltrato en la adultez a manos de futuras parejas y asumirlas como normales. Lo que conlleva a que se perpetúe este tipo de problemas en la sociedad, sin embargo, las costumbres y cultura de cada familia influencia de manera directa en el comportamiento de la persona.

Las mujeres que han sufrido violencia en su infancia o crecieron en un ambiente de tensión o de peligro, tienden a dejarse manipular por sus parejas y como consecuencia a nivel social se puede decir que: "El hecho de presenciar la violencia doméstica crónica puede marcar la iniciación de una pauta de delincuencia y uso de la violencia en las relaciones personales que durará toda la vida..." (Presidente Constitucional de la República, 2007)

\subsection{Dependencia económica}

Según Rey (2002) en un estudio realizado en Colombia expone que: "Una buena proporción de las mujeres víctimas de violencia conyugal poseen un bajo nivel educativo y provienen de familias de escasos recursos económicos, por lo que sus oportunidades de desarrollo socioeconómico 
son reducidas" esta situación pone a la mujer en riesgo al depender únicamente de su pareja.

Según Rey (2002) afirma: "En relación con el estado civil, se ha encontrado dos situaciones: la mujer víctima lleva bastante tiempo casada o en unión libre, o es joven y se ha separado recientemente de su pareja, en buena parte motivada por la situación de violencia", lo que afirma que las mujeres no denuncian estos hechos de violencia con brevedad, sino más bien perduran años con el maltratador, también expone que: "Existe una mayor frecuencia de violencia familiar en las zonas urbanas" (Rey, 2002). De acuerdo a esta expresión la violencia intrafamiliar no solo afecta a determinadas zonas, sino que más bien está inmersa en la sociedad en general.

\subsection{Alcoholismo y drogadicción}

Según Herrera Paredes \& Arena Ventura (2010) expresan que: "La violencia y el maltrato familiar se han reconocido como un problema socialmente vinculado al consumo de alcohol, principalmente consumido por el hombre, quien ejecuta algún tipo de violencia en la mujer". De lo expresado se puede evidenciar que el consumo de alcohol influye en la violencia intrafamiliar más no se puede decir, que sea la causa para que se propague.

Sin embargo, la relación que existe entre el consumo de alcohol y la violencia intrafamiliar ha constituido un problema a nivel social, según Herrera Paredes \& Arena Ventura (2010), afirman:

El inicio temprano del consumo de alcohol o drogas, el contacto con padres alcohólicos o drogadictos en la etapa infantil, la existencia de abusos sexuales en la niñez, el maltrato entre padres, los resentimientos por una infancia traumática y el status económico, conllevan al maltrato físico y psíquico hacia la familia.

\subsection{Violencia y maltrato}

Durante la infancia se aprenden diferentes pautas de comportamiento y se asimilan algunas conductas, quienes han observado dentro de su núcleo familiar algunas conductas agresivas o situaciones de violencia, pueden generar estos comportamientos a futuro con su pareja, según Rey (2002) expresa: "El maltrato en la familia de origen puede facilitar el aprendizaje de pautas inadecuadas de relación que después son aplicadas con la propia pareja en la edad adulta".

En un estudio acerca de la violencia física contra la mujer menciona: "En cuanto a experiencias en su familia de origen, 64, $4 \%$ de las mujeres indicaron haber recibido algún tipo de maltrato durante su niñez" (Klevens, 2001). Lo que significa que las víctimas de violencia en la niñez tienden a sufrir este tipo de maltrato en la etapa conyugal.

\subsection{Violencia intrafamiliar}

Es importante mencionar que: "La violencia intrafamiliar se define como aquella que tiene lugar dentro de la familia ya sea que el agresor comparta o haya compartido el mismo domicilio y que comprende, entre otros, violación, maltrato físico, sicológico y abuso sexual. (?, ?)Núñez de Arco \& Carvajal, 2004). Lo que se entiende que existen diferentes tipos de violencia hacia la mujer dentro de la sociedad, sin embargo, aún en la actualidad no se reconocen tales tipos de violencia, pues las mujeres siguen contemplando que solo el maltrato físico sería tomado como violencia, debido a factores sociales y culturales que han sido trasmitidos de generación en generación y aún no se han logrado romper pues persiste la imagen de la mujer como sumisa y débil comparado al hombre. Otro tipo de violencia establecido es aquella que se trata de la patrimonial, que tiene que ver con la prohibición del uso del dinero propio de la persona o destrucción de materiales de valor para la mujer por parte del hombre, en la actualidad las mujeres no asumen los diferentes tipos de agresiones como violencia hacia ella.

\section{Metodología}

Para el abordaje de la investigación se utilizó el método mixto (cuali-cuantitativo) no experimental, reflejado directamente en encuestas estructuradas, entrevistas personales y un cuestionario. La investigación permitió comprender de manera amplia y profunda el fenómeno estudiado; la naturaleza descriptiva, por tanto, buscó narrar los factores socioculturales que influyen en mujeres que han sufrido violencia intrafamiliar.

En la Casa de Acogida "María Amor" se trabajó con el universo de la población, es decir 12 mujeres que se encuentran en situación de acogida, así también en el Centro de Atención Integral en casos de violencia intrafamiliar y/o sexual "Corporación Mujer a Mujer", la muestra tomada fue aleatoria, trabajando con 15 mujeres seleccionadas, contando con una población total de 27 mujeres.

\section{Resultados}

Del proceso de recolección y análisis de la información se generaron los siguientes datos identificativos de la población estudiada.

El 22,22\% de mujeres encuestadas se encuentran entre los 26-30 años de edad mientras que el 18, $52 \%$ están entre los 21-25 años, con el mismo porcentaje de 18,52\% se encuentran las mujeres de 41-45 años y tan solo el $3.70 \%$ de 15-20 año. El 66, $67 \%$ de las personas encuestadas practican la religión católica, mientras que el 22, $22 \%$ corresponden a otro tipo de religión no especificada.

El 59,26\% de mujeres viven en el área urbana del Cantón Cuenca, mientras que el 40, $74 \%$ corresponden al área rural. El 51, $85 \%$ de las mujeres relejan un estado civil soltera, el $29,63 \%$ casada, el $14 \%$ se encuentra divorciada y el 3, 70\% se encuentra en unión libre. El 55, 56\% de mujeres, únicamente han cursado la primaria, mientras que el 33, 33\% cuenta con estudios secundarios y el 11, $11 \%$ reflejan un nivel de instrucción superior. Con respecto a la vivienda, el 48, 15\% de las mujeres habita en casas 
arrendadas, el 33, $33 \%$ tiene casa propia, y el 18, 52\% habitan en casas prestadas.

El 92, 59\% de las mujeres se auto identifican como mestizas, mientras que el 7, 41\% se consideran blancas. Del total de la población encuestada el 96, 30\% tienen cargas familiares (hijos) y el 3, 70\% no. La cantidad de hijos se presenta con iguales porcentajes entre uno, tres y cuatro hijos $(29,63 \%)$, y el $11,11 \%$ de mujeres tienen dos hijos. El 48, $15 \%$ no trabaja, por lo tanto, carece de un ingreso mensual, mientras que el 33,33\% tiene un ingreso mensual de 100-200 y tan solo el 7,41\% de mujeres percibe un sueldo mensual de entre $301-400$.

El 51, $85 \%$ de mujeres refieren que los problemas o conflictos de su familia en la niñez se resolvían a través de los golpes. El 44, $44 \%$ de mujeres encuestadas declaran haber presenciado actos de violencia en su entorno social, mientras que el 29, $63 \%$ nunca lo ha presenciado. El 66, $67 \%$ de mujeres refieren que su madre fue la víctima más frecuente de violencia en el hogar.

El 51, $85 \%$ manifiesta haber sido víctima de violencia física en su infancia.

Tabla 1 Violencia Física

\begin{tabular}{|l|r|r|r|r|r|}
\hline $\begin{array}{c}\text { Etiqueta de } \\
\text { Valor }\end{array}$ & Valor & Frecuencia & Porcentaje & $\begin{array}{c}\text { Porcentaje } \\
\text { Válido }\end{array}$ & $\begin{array}{c}\text { Porcentaje } \\
\text { Acumulado }\end{array}$ \\
\hline \hline FÍSICA & 1 & 18 & 66,67 & 100,00 & 100,00 \\
\hline &. & 9 & 33,33 & Perdidos & \\
\hline & Total & 27 & 100,0 & 100,0 & \\
\hline
\end{tabular}

Fuente: Casa de Acogida "María Amor" y Centro de atención "Corporación Mujer a Mujer"

Figura 1. Violencia Física

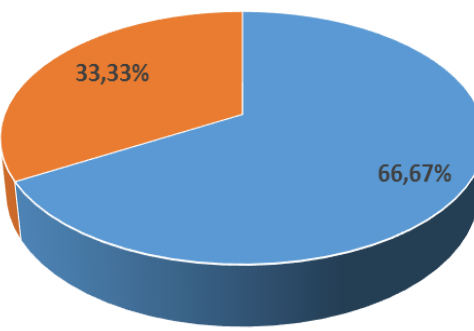

- FÍSICA - SIN RESPUESTA

Fuente: Casa de Acogida "María Amor" y Centro de atención "Corporación Mujer a Mujer"

El 66, $67 \%$ de mujeres encuestadas manifiestan haber sufrido violencia física por parte de su ex pareja, la mayoría de mujeres encuestadas han sido víctimas de violencia física por parte de su pareja, de no tomar en cuenta este tipo de violencia como el más peligroso en la actualidad se seguiría aumentando la lista de femicidios en el Ecuador que según Víctor Aráus jefe de la DINASED (2017) "se han registrado 85 casos de femicidios hasta septiembre del presente año".
Tabla 2 Violencia Psicológica

\begin{tabular}{|c|r|r|r|r|r|}
\hline $\begin{array}{c}\text { Etiqueta de } \\
\text { Valor }\end{array}$ & Valor & Frecuencia & Porcentaje & $\begin{array}{c}\text { Porcentaje } \\
\text { Válido }\end{array}$ & $\begin{array}{c}\text { Porcentaje } \\
\text { Acumulado }\end{array}$ \\
\hline PSICOLÓGICA & 2 & 25 & 92,59 & 100,00 & 100,00 \\
&. & 2 & 7,41 & Perdidos & \\
\hline \hline & Total & 27 & 100.0 & 100.0 & \\
\hline
\end{tabular}

Fuente: Casa de Acogida "María Amor" y Centro de atención "Corporación Mujer a Mujer"

Figura 2. Violencia Psicológica

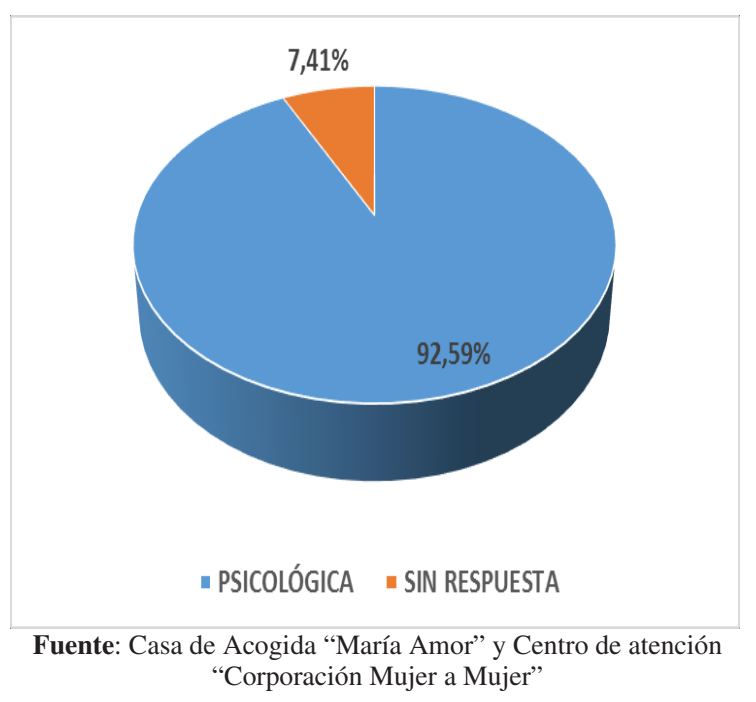

El 92, 59\% de mujeres encuestadas indican haber sufrido violencia psicológica por parte de su ex pareja. La mayor parte de mujeres expresa que antes de conocer que existe este tipo de violencia, la asimilaba como algo normal dentro del hogar, pero al manifestarse de forma repetitiva las sensibiliza y las lleva a vivir situaciones de desvalorización personal.

Tabla 3 Violencia Sexual

\begin{tabular}{|l|r|r|r|r|r|}
\hline $\begin{array}{c}\text { Etiqueta de } \\
\text { Valor }\end{array}$ & Valor & Frecuencia & Porcentaje & $\begin{array}{c}\text { Porcentaje } \\
\text { Válido }\end{array}$ & $\begin{array}{c}\text { Porcentaje } \\
\text { Acumulado }\end{array}$ \\
\hline SEXUAL & 3 & 11 & 40,74 & 100,00 & 100,00 \\
\hline \hline & Total & 27 & 100,0 & 100,0 & \\
\hline
\end{tabular}

Fuente: Casa de Acogida "María Amor" y Centro de atención "Corporación Mujer a Mujer" 
Figura 3. Violencia Sexual

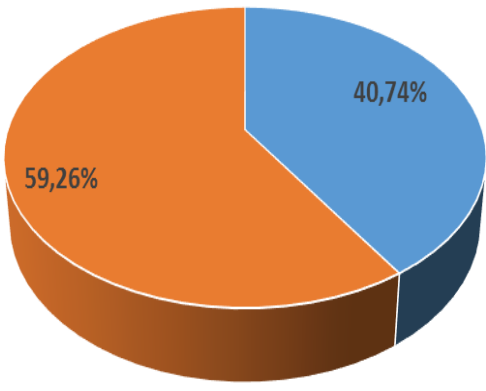

- SEXUAL — SIN RESPUESTA

Fuente: Casa de Acogida "María Amor" y Centro de atención "Corporación Mujer a Mujer"

El $40,74 \%$ de las encuestadas expresa haber sufrido violencia sexual por su ex pareja, sin embargo, se debe desmerecer la naturalización de la violencia, más aun dentro del seno del hogar por lo que las mujeres siguen creyendo que al ser su pareja deben someterse a ciertas prácticas sexuales aunque no estén de acuerdo.

Tabla 4 Violencia Patrimonial

\begin{tabular}{|c|r|r|r|r|r|}
\hline $\begin{array}{c}\text { Etiqueta de } \\
\text { Valor }\end{array}$ & Valor & Frecuencia & Porcentaje & $\begin{array}{c}\text { Porcentaje } \\
\text { Válido }\end{array}$ & $\begin{array}{c}\text { Porcentaje } \\
\text { Acumulado }\end{array}$ \\
\hline PATRIMONIAL & 4 & 12 & 44,44 & 100,00 & 100,00 \\
& $\cdot$ & 15 & 55,56 & Perdidos & \\
\hline \hline \multicolumn{2}{r|}{ Total } & 27 & 100,0 & 100,0 & \\
\hline
\end{tabular}

Fuente: Casa de Acogida "María Amor" y Centro de atención "Corporación Mujer a Mujer"

Figura 4. Violencia Patrimonial

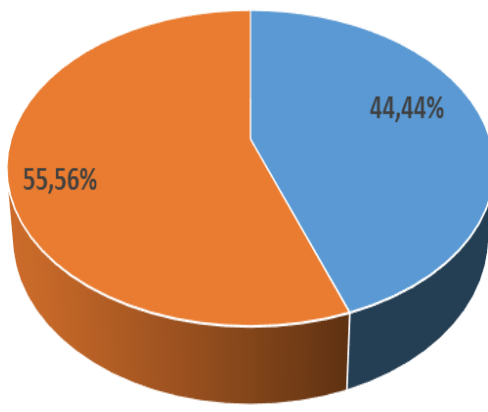

\section{PATRIMONIAL - SIN RESPUESTA}

Fuente: Casa de Acogida "María Amor" y Centro de atención "Corporación Mujer a Mujer"

Del total de la población encuestada el 44, $44 \%$ expresa haber sufrido violencia patrimonial por parte de su ex pareja, sin embargo expresan que no conocen a profundidad sobre este tipo de violencia, lo que las lleva a la ignorancia al momento de describir esta violencia, necesitando brindar información acerca de este tipo violencia. Según Flores \& Espejel (2012): "El concepto de violencia patrimonial se refiere a cualquier acto u omisión que afecte la supervivencia de la víctima".

Tabla 5 Violencia Dependencia económica

\begin{tabular}{|l|r|r|r|r|r|}
\hline Etiqueta de Valor & Valor & Frecuencia & Porcentaje & $\begin{array}{c}\text { Porcentaje } \\
\text { Válido }\end{array}$ & $\begin{array}{c}\text { Porcentaje } \\
\text { Acumulado }\end{array}$ \\
\hline DEPENDENCIA & 2 & 14 & 51,85 & 100,00 & 100,00 \\
ECONÓMICA & & 13 & 48,15 & Perdidos & \\
\hline & Total & 27 & 100,0 & 100,0 & \\
\hline
\end{tabular}

Fuente: Casa de Acogida "María Amor" y Centro de atención "Corporación Mujer a Mujer"

Figura 5. Violencia Dependencia económica

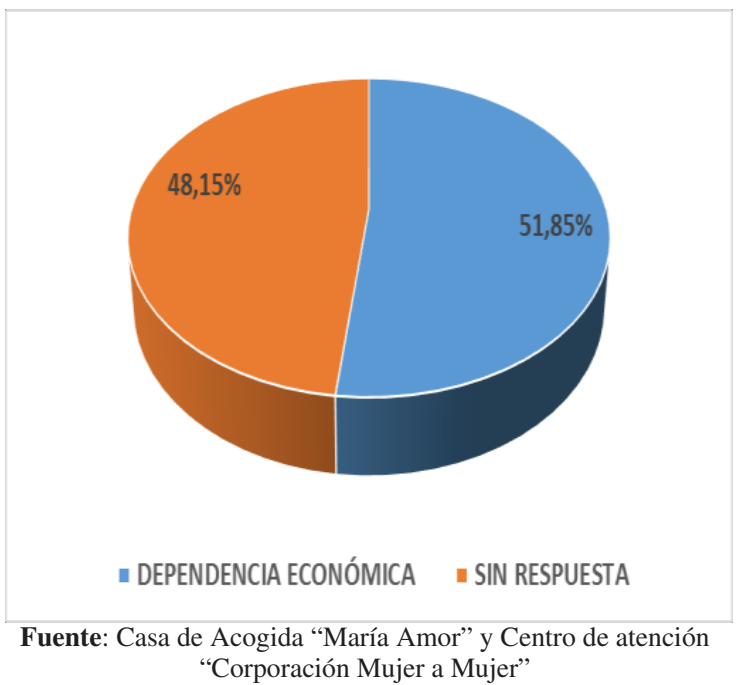

El 51, $85 \%$ de mujeres manifiesta que permanecen con el maltratador por dependencia económica. Las mujeres estarían sujetas a vivir cualquier tipo de maltrato por esta razón, lo que indica la importancia de implementar un plan guiado a elevar las capacidades de las personas, para que sean útiles para sí mismas y para la sociedad, de esta manera se estaría evitando la violencia intrafamiliar.

\subsection{Análisis e Interpretación}

Se evidencia que la mayoría de las mujeres no se separa de su maltratador por temor a quedarse sola, pues la presión hacia lo que los demás piensen de una persona al separarse de su pareja es demasiado inminente aun en la actualidad, lo que predispone a la mujer a convivir con su pareja por mucho tiempo en relaciones toxicas.

Del análisis realizado se establece que la costumbre no es un factor determinante para las mujeres a la hora de decidir quedarse con la persona que la maltrata (33, $33 \%$ ), según Camas (2010), la violencia intrafamiliar desde una perspectiva social expone que: "Hay evidencia de que 
los aspectos sociales jueguen papeles importantes en la expresión de las conductas violentas, uno de ellos es la trasmisión intergeneracional de la violencia".

Del universo de la población, el 59, $62 \%$ de las mujeres dicen que la razón para quedarse junto al maltratador es por los hijos, pues la necesidad de que los menores crezcan a lado del padre se vuelve fundamental en los ideales de las mujeres. Así también, del total de la población el 59, $26 \%$ manifiesta no haber buscado ayuda inmediata cuando comenzó a sufrir violencia. Posteriormente el 70, $37 \%$ de mujeres expresa haber acudido a servicios de apoyo a la mujer, el 11,11\% acudieron al juzgado de la familia, el $7,41 \%$ acudieron a la policía y tan solo el $3,70 \%$ a la fiscalía.

Con la utilización del estadígrafo Chi cuadrado (X2), se determinó la relación entre los factores socioculturales y la violencia intrafamiliar en las mujeres que acuden a los centros de apoyo a la mujer "María Amor" y el centro de atención "Corporación Mujer a Mujer" del cantón Cuenca. De tal forma que del universo de la población, el 44,44\% que han presenciado violencia durante su infancia y han buscado ayuda en su etapa adulta evidencian porcentajes significativos con los factores socioculturales analizados.

\section{Conclusiones}

Las mujeres encuestadas practican mayormente la religión católica, lo que concuerda con las creencias culturales como la expresión "En mi familia nos han enseñado a que si te casas es para siempre", exponiendo a la mujer a sufrir violencia por parte de su pareja al estar sometida a este tipo de creencias o mitos durante su vida.

Dentro del estudio se verifica que la violencia intrafamiliar no varía según del área de residencia de la persona, por lo que se establece que esta se puede dar en cualquier zona de residencia.

La mayor parte de mujeres encuestadas manifiesta haber sufrido algún tipo de violencia por parte de su pareja, sin embargo, permanecen a su lado por diferentes motivos, los más comunes según los resultados obtenidos son: "dependencia económica", "temor a quedarse sola", y "por los hijos". Lo que implica establecer un trabajo dentro de estos tres ítems evitando así propagar la violencia en los hogares.

El 51,85\% del total de mujeres encuestadas establecen que sus agresores en la infancia consumían alcohol, convirtiéndose en un desencadenante de violencia intrafamiliar, sin embargo, no se le puede atribuir directamente a este factor como el principal riesgo dentro de la familia.

\section{Recomendaciones}

Se recomienda establecer un plan de intervención en cuanto a la reeducación de las mujeres, evitando la propagación de creencias y mitos que aún persisten en la actualidad, Elaborando campañas y talleres de prevención que busquen romper con los patrones socioculturales eliminando cualquier tipo de violencia contra las mujeres.
Se recomienda establecer planes de mejora en cuanto a la economía del hogar y el empoderamiento económico de la mujer, es decir elevar las capacidades y que sean ellas participes de su propio cambio a partir de su concientización y revalorización como personas activas y sujetas de derechos.

Concientización a la población en general, sobre la violencia intrafamiliar y sus tipos, buscar llegar a las comunidades rurales del Cantón, dar a conocer a las mujeres sus derechos y centros de apoyo, en caso de pasar por situaciones de violencia. Para ello es imprescindible que, a conciencia de que la violencia no es natural al ser humano y responde a patrones socioculturales naturalizados, única y exclusivamente la educación podrá erradicar este problema social que cobra vidas humanas

\section{Referencias Bibliográficas}

Equipo del departamento de planificación y gestión para la equidad social y de Género. (2016). Mira distinto, actúa diferente. Cuenca: Servigraf.

Espinoza Morales, M., Alazales Javiqué, M., Madrazo Hernández, B., García Socarrás, A. M., y Presno Lavrador, M. C. (2001). Violencia intrafamiliar, realidad de la mujer latinoamericana. SCIELO.

Herrera Paredes, J. M., y Arena Ventura, C. A. (2010). Consumo de alcohol y violencia doméstica contra las mujeres: un estudio con estudiantes universitarias de México. México.

Klevens, J. (2001). Violencia física contra la mujer en Santa Fé de Bogotá: prevalencia y factores asociados. Panam Salud Pública.

Labrador, F. J., Paz Rincón, P., Luis, P., y FernándezVelasco, R. (2004). Mujeres víctimas de violencia doméstica. Madrid: Pirámide.

Municipalidad de Cuenca. (2015). Plan de desarrollo y ordenamiento territorial del Cantón Cuenca. Cuenca: Municipalidad de Cuenca.

Presidente Constitucional de la República. (2007). Plan Nacional de Erradicación de Violencia de Género. Quito: Presidencia Nacional.

Quintana Zurita, J., Serrano Salgado, J., Pimentel Bolaños, Y., José Luis, y Moncayo, R. (2014). La violencia de género contra las mujeres en el Ecuador. Quito.

Rey, C. (2002). Rasgos sociodemográficos e historia de maltrato en la familia de origen, de un grupo de hombres que han ejercido violencia hacia su pareja y de un grupo de mujeres víctimas de este tipo de violencia. Revista Colombiana de Psicología, 8190.

Ruiz-Perez, I., Blanco-Prieto, P., y Vives-Cases, C. (2004). Violencia contra la mujer en la pareja: determinantes y respuestas sociosanitarias. SCIELO.

Sagot, M. (2000). Ruta Crítica de las mujeres afectadas por la vioencia intrafamiliar en América Latína. 
Soledispa Toro, A., y Garbay Mancheno, S. (2004). Manual de Atención Legal en casos de violencia Intrafamiliar y de Género. Quito: FES DIS / CEPAM.

Recibido: 30 de junio de 2018

Aceptado: 3 de septiembre de 2018 
